\title{
Effects of a multidisciplinary quality of life intervention on sleep quality in patients with advanced cancer receiving radiation therapy
}

\section{Melanie T. Gentry ${ }^{1}$, Pamela J. Atherton ${ }^{2}$, Maria Lapid1, Simon Kung ${ }^{1}$, Shezad K. Niazi ${ }^{3}$, William V. Bobo ${ }^{3}$, Jarrett. Richardson', Matthew M. Clark1, Teresa A. Rummans ${ }^{1}$ \\ ${ }^{1}$ Mayo Clinic, Psychiatry and Psychology, Rochester- MN, USA. ${ }^{2}$ Mayo Clinic, Division of Biomedical Statistics and Informatics- Department of Health Sciences, Rochester, USA. ${ }^{3}$ Mayo Clinic, Psychiatry and Psychology, Jacksonville, USA.}

\section{Background}

Sleep disturbances are quite prevalent in cancer patients, especially for those with advanced disease.[1] Most intervention studies to improve sleep in cancer populations have been in early stage cancer patients. There are few psychosocial or pharmacological intervention studies to address sleep issues in patients with advanced cancer or receiving active cancer treatment. [2]

\section{Study Aims}

This study assesses the impact of a multidisciplinary quality of life (QOL) intervention on subjective sleep difficulties in patients with advanced cancer.

\section{Methods}

A multidisciplinary intervention targeting five domains of QOL (physical, mental, social, spiritual, and emotional) was developed for patients with advanced cancer receiving radiation therapy. [3]

- 117 patients were enrolled and were randomly assigned to intervention $(n=54)$ or standard care $(n=63)$.

The intervention group attended six intervention sessions while the standard care group received informational material only.

- Questionnaires to assess sleep difficulties and daytime sedation, the Pittsburgh Sleep Quality Index (PSQI) and Epworth Sleepiness Scale (ESS), were administered at baseline, and weeks 4 (post intervention), 27 and 52 .

\begin{tabular}{|r|r|r|r|}
\multicolumn{4}{|c|}{ Patient Population } \\
Age & & Gender & \\
Mean (SD) & $59.8(11.0)$ & Female & $39(33.3 \%)$ \\
Median & 60.0 & Male & $78(66.7 \%)$ \\
\hline Performance Score & & Planned Treatment & \\
Fully Active & $62(53.0 \%)$ & Radiation & $18(15.4 \%)$ \\
Restricted & $52(44.4 \%)$ & Chemotherapy & $1(0.9 \%)$ \\
Ambulatory & $3(2.6 \%)$ & Both & $98(83.8 \%)$ \\
\hline Dominant Disease & & Tumor & \\
Status & $25(21.4 \%)$ & Grade & $13(11.1 \%)$ \\
Brain & $17(14.5 \%)$ & $1-2$ & $50(42.7 \%)$ \\
Head \& Neck & $17(14.5 \%)$ & 4 & $35(29.9 \%)$ \\
Lung & $44(37.6 \%)$ & 5 & $19(16.2 \%)$ \\
GI & $14(12.0 \%)$ & & \\
Other & & &
\end{tabular}

\section{Results}

The intervention group reported better PSQI sleep quality than the control group at week $4(p=0.04)$. Although both groups showed improvements in sleep measures from baseline, there were no statistically significant differences between groups in any of the PSQI component scores, PSQI total score or ESS at week 27. At week 52, the intervention group used less sleep medication than control patients compared to baseline $(p=0.04)$.

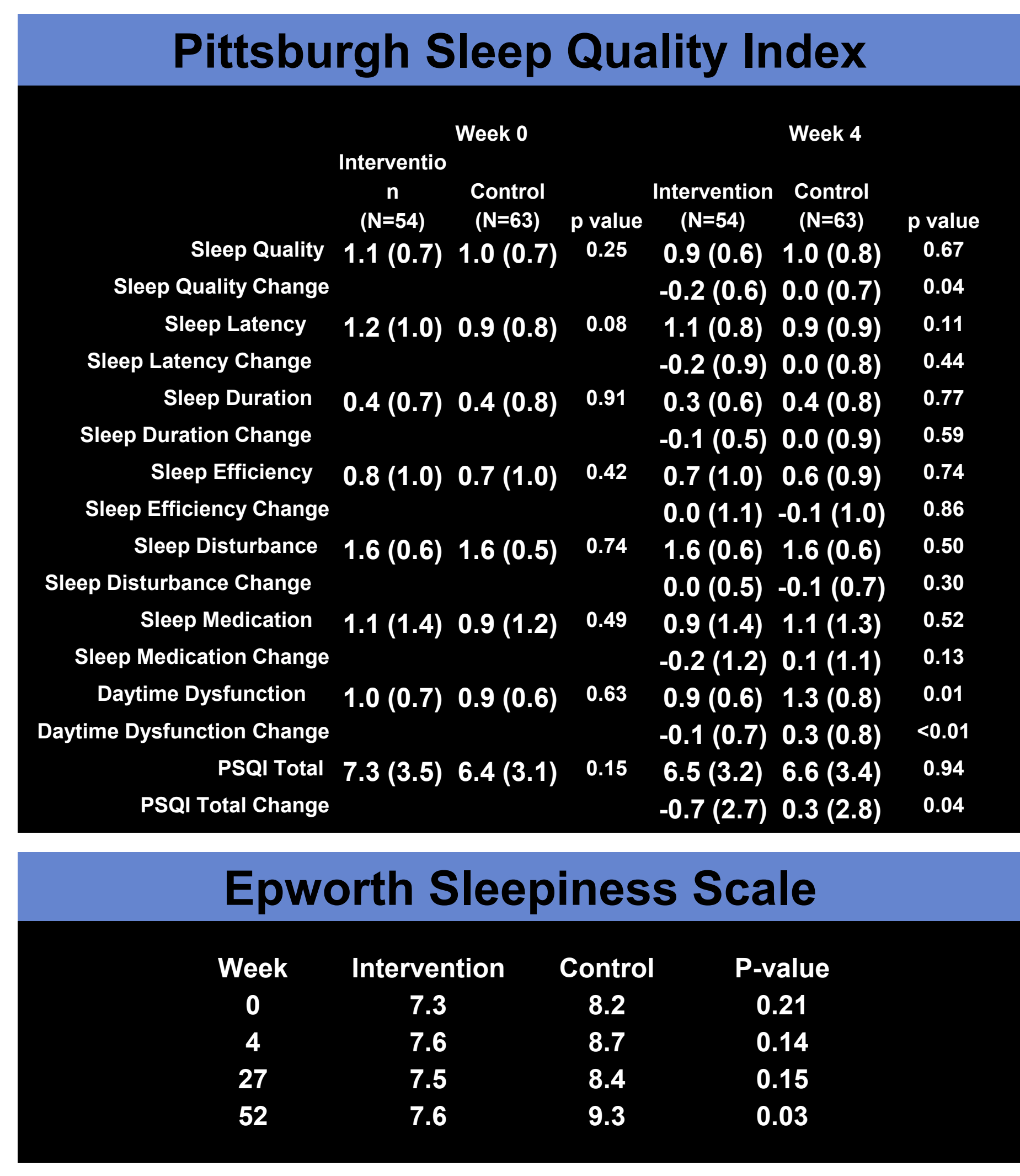

\section{Conclusions}

- This intervention helped improved some domains of subjective sleep quality of cancer patients undergoing radiation therapy.

Given the multifactorial etiology of sleep disturbances and the profound need for sleep intervention studies in advanced cancer patients, future clinical trials should incorporate multidisciplinary interventions to address QOL, sleep quality and their impact on long term survival.

\section{References/Contact}

Clark, J., Cunningham, M., McMillan, S., Vena, C. \& Parker, K (2004). Sleep-wake disturbances in people with cancer part II: evaluating the evidence for clinical decision making. Oncology Nursing Forum, 31(4), 747-771.

Mystakidou, K., Parpa, E., Tsilika, E., Gennatas, C., Galanos, A. \& Vlahos, L. (2009). How is sleep quality affected by the psychological and symptom distress of advanced cancer patients? Palliative Medicine, 23(1), 46-53.

Clark, M.M., Rummans, T.A., Atherton, P.J., Cheville, A.L., Johnson, M.E., Frost, M.H., Miller, J.J., Sloan, J.A., Graszer, K.M., Haas, J.G., Hanson, J.M., Garces, Y.I., Piderman, K.M. Lapid, M.I., Netzel, P.J., Richardson, J.W. \& Brown, P.D. (2013

Contact: Melanie.gentry@mayo.edu 